\title{
THE USE OF FUZZY NEURAL STRUCTURES TO INCREASE THE Reliability of Drilling Platforms
}

\author{
Nyrkov Anatoliy, Sergei Chernyi, Anton Zhilenkov, Sergey Sokolov \\ Admiral Makarov State University of Maritime and Inland Shipping, Saint Petersburg, 198035, Russian Federation \\ Kerch State Maritime Technological University.82, Ordzhonikidze St., Kerch, 298309, Russian Federation
}

\begin{abstract}
The use of semiconductor converters as a part of sea drilling platforms' electrical power systems is the cause of rising of electromagnetic compatibility and inactive power problems that demands an incorporation of the active filter compensation devices. It is known that in such real systems the capacitor of filter is discharging because of the existence of active power losses and it has negative impact on electric power quality.

In the article, authors propose advanced ANFIS controller that allows to reduce the maximum voltage deviation on capacitor bank, and to reach of the established voltage value within several periods of circuit voltage. The suggested controller was modeled in the ANFIS editor of the MATLAB software environment. It is shown that by mean of number of proposed innovations the consumed computing power of the digital system realizing the ANFIS controller decreased in dozens of times in comparison with regular fuzzy logic controller implementation.
\end{abstract}

Keywords: autonomous energy system; active harmonic conditioner; voltage deviation; fuzzy logic; artificial neuron network
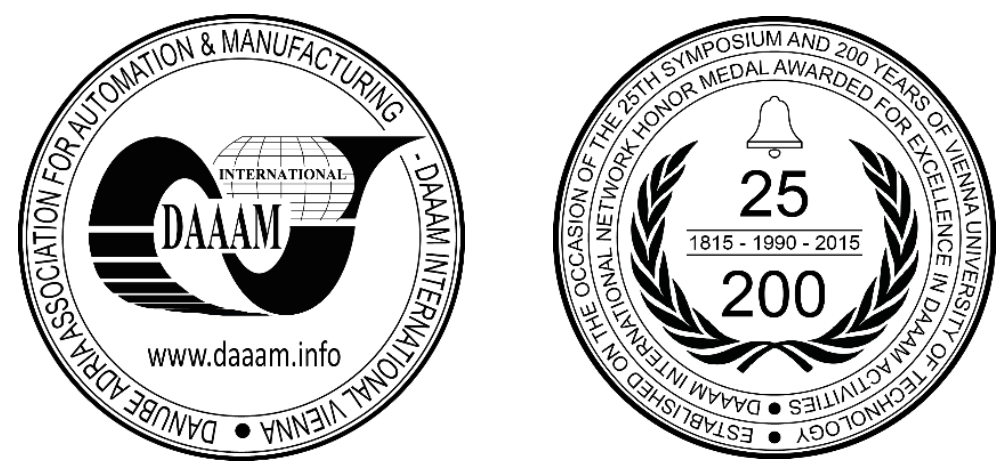

This Publication has to be referred as: Nyrkov, A[natoliy]; Chernyi, S[ergei]; Zhilenkov, A[nton] \& Sokolov, S[ergey] (2016). The use of Fuzzy Neural Structures to Increase the Reliability of Drilling Platforms, Proceedings of the 26th DAAAM International Symposium, pp.0672-0677, B. Katalinic (Ed.), Published by DAAAM International, ISBN 9783-902734-07-5, ISSN 1726-9679, Vienna, Austria DOI: $10.2507 / 26$ th.daaam.proceedings.091 


\section{Analysis of the problem. General aspects.}

The use of semiconductor converters for main drive's power supply as a part of sea drilling platforms' electrical power systems (EPS) is the reason of rising problems of equipment's electromagnetic compatibility in EPS and a loading with an inactive power of autonomous power supply. These devices are used as frequency converters for alternating current motors or the controlled rectifiers in direct current systems. High reactive currents and a wide band of the circuit currents' higher harmonics and voltage (which amplitude as a rule exceeds admissible by the proscriptive documents value) created by semiconductor converters, demand an incorporation of the filter compensation devices (FCD).

The searches of decisions on optimization of FCD are continuing nowadays, both in the context of power part, and in context of their control systems. It should be noted that specific requirements are imposed to FCD applied on such autonomous objects as sea drilling platforms at commensurability of the loading powered via semiconductor converters with a lack of circuit's neutral conductor (isolated neutral system), an instability of electric power circuit key parameters, etc.

The active FCD (AFCD) combining the reactive power compensators (RPC) and the active filters (AF) of harmonics constructed, as a rule, on IGBT transistors are promising here. The subject development of active FCD or AFCD new control systems was studied in a number of works and the solution was found, in particular, and in the works of this article's authors [2-3], however, much less of the published works raise the subject of AFCD stability research.

It is known that the output signal of the AFCD converter is voltage modulation of the energy storage unit as which the reservoir capacitor or the capacitor bank (CB), as a rule acts. Ideally the compensator operating as a part of RPC or AF doesn't consume and doesn't generate active power and, as a result, an average value of voltage on the capacitor bank doesn't change, therefore, the additional power supply on the side of AF direct current isn't required. But in real system (actual system) the capacitor will discharge because of the existence of active power losses in AFCD.

We will consider typical structure of this block. The connection of converter output to filter chokes on the side of alternating current is shown on the flowchart of fig. 1. On the side of a direct current the reservoir capacitor $\mathrm{C}$ is connected parallel to which voltage sensor is connected. Voltage and circuit current sensors are shown on the flowchart, current sensors in filter throttles. Signals from sensors arrive on control system input, and the output of a control system is connected to voltage converter driver. Signals from the driver arrive on the operating input of transistors of the converter.

\section{A structural representation of the problem domain}

Voltage deviation on CB from nominal value caused by active losses in the compensator. A voltage change of EPS or jump of load value reflect the influence of these factors on voltage on CB. This will cause the change of voltages $u_{a f}, u_{b f}, u_{c f}$ at the compensator output, and, therefore, the compensating currents also generated by it. That will cause a control error. On the other hand, voltages $u_{a f}, u_{b f}, u_{c f}$ depend obviously on $u_{d c}$ and switching functions $f_{a}, f_{b} u f_{c}$ of semiconductor switches. However, and $u_{d c}$ is a dependence on the switching functions and inactive currents generated by the compensator.

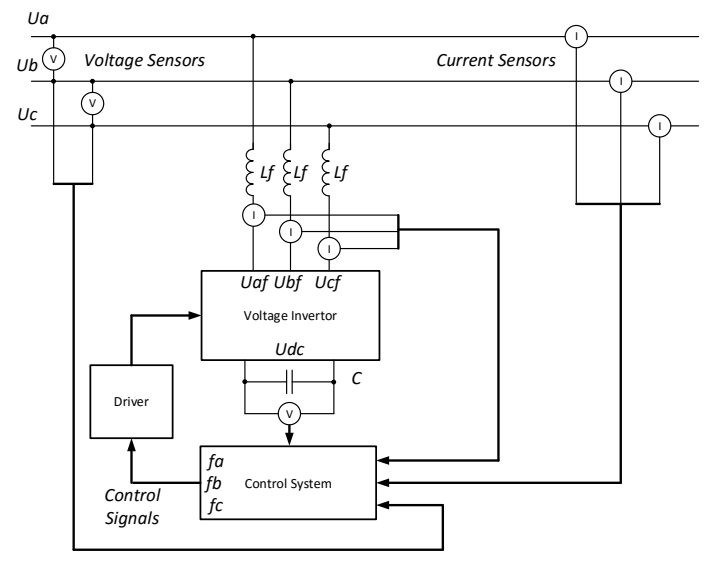

Fig. 1. The flowchart of AFCD.

Besides a steady state operation of the compensator (in which there is an exchange of inactive current between a circuit and the compensator) two operating options concerning CB voltage are possible: when voltage on CB exceeds preset one and when it is less than preset. In the first case voltage change $u_{a f}, u_{b f}$ and $u_{c f}$, can be prevented operating the switching functions $f_{a}, f_{b}$ и $f_{c}$, that is to carry out regulation only on compensating current, reducing the output voltage of the compensator. However voltage on $\mathrm{CB}$ can exceed admissible value at such regulation that will lead to destruction of capacitors. Besides, it is impossible to compensate the uncontrolled reduction of compensating current at voltage reduction on $\mathrm{CB}$ lower than the preset one in such a way. 
As a result, the changes of voltage on $\mathrm{CB}$ need to be compensated at the expense of consumption from the alternating current circuit equal active power. It is possible to embody it by an introduction of a compensator's CB voltage regulator to a control system with a feedback between voltage on the capacitor and filter's current. As a result, filter's current will contain a necessary active component which value is established by the losses of active power in compensator's elements which, in their turn, are established by: $\quad$ a current value;

- a switching frequency of switching devices

- $\quad$ an admissible deviation value of filter's current from a reference value;

- an average value of voltage on the capacitor;

- $\quad$ an inductance of the matched filter (matching filter).

A controller has to add such component into a signal of pedestal current that dc-to-ac converter consumed current in phase to the first harmonic. That is to consume a charge current of $\mathrm{CB}$ in phase an active component of circuit current to reduce voltage fluctuations on the capacitor and to compensate the losses of active power in FCD at voltage reduction on $\mathrm{CB}$.

\section{The design of a CB voltage controller on a fuzzy logic}

In the majority of solutions voltage controller on the AFCD capacitor represents the PI controller which parameters are determined from a linearized mathematical model of a control object. However, a need for parameters tuning of PI controller arises at the parameter change of control object that takes time, the corresponding test and measuring equipment and, as a rule, is impossible in the course of FCD use. Moreover, the transients of similar control systems are characterized by the inadmissible values of overshoot and generally, a control law in similar systems is nonoptimal. At the same time, voltage deviations on CB bring distortions in the AFCD compensating current, reducing the provided power quality parameter $[1,2]$.

In this study the controller on the basis of the fuzzy logic (FL) is offered as an alternative to the PI-controller. The block diagram of controller on FL is given in fig. 2. Two input signals were used for its construction:

-errors (a difference between the pedestal voltage and the actual voltage on $\mathrm{CB}$ ) $-e$;

- the changes of error's signal for one sampling period (a difference between the previous and current value of error signal) $-\Delta e$.

As a result of simulation it was established that the best and sufficient (on a ratio of FL controller's quality and complexity) variant is the use of seven membership functions with the following linguistic values $\bullet \quad$ NB $\quad-\quad$ the greatest negative;

- $\quad \mathrm{NM}$ - an average negative;

- $\quad \mathrm{SM}$-a small negative;

- ZE - zero;

- $\quad$ PS - a positive small;

- $\quad \mathrm{PM}$ - a positive average;

- $\quad$ PB - a positive big.

The triangular membership functions are, as a rule, used at the construction of FL-systems that is caused generally by a low value of the required computational capability for its introduction. However, it is obvious that a robustness of functions with such form - low as their central value is actually presented by a point. According to researches of article's authors, Gaussian copula functions possess the best qualities from the point of view of a robustness, and the worst - $t-$ function.

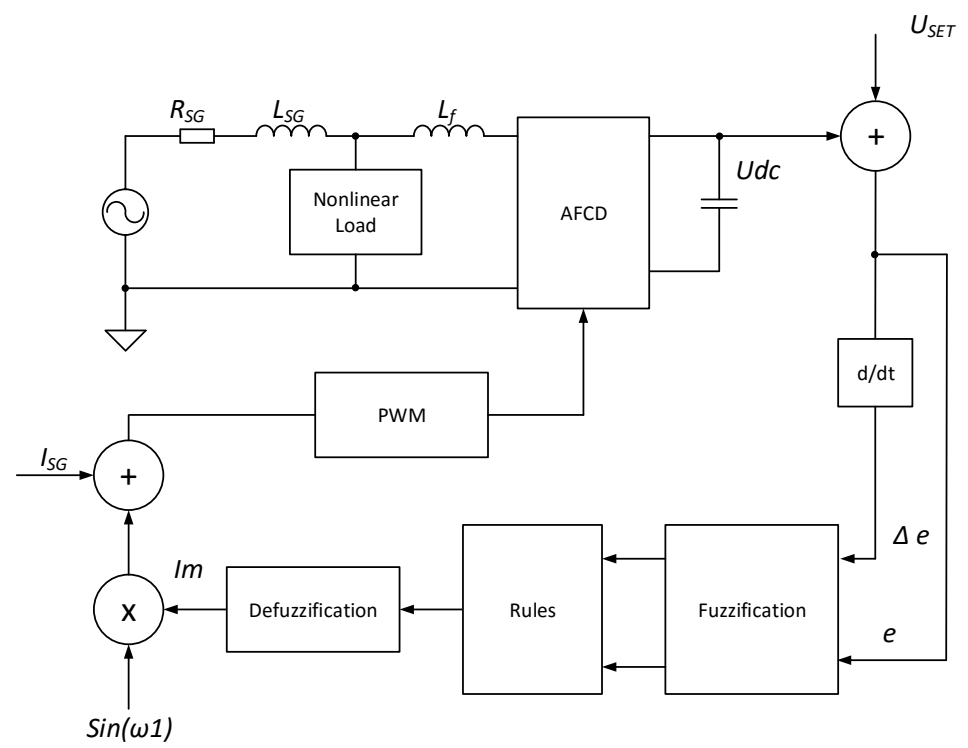

Fig. 2.The structure of the AFCD FL-controller. 
Seven fuzzy sets with membership functions similar in a form to the functions of the fuzzification block and the representing following linguistic values are formed at the output of a defuzzification block:

- $\quad \mathrm{NOB}$ - the greatest negative;

- $\mathrm{NOM} \mathrm{-} \mathrm{an} \mathrm{average} \mathrm{negative;}$

- $\mathrm{SOM}$ - small negative;

- ZEO - zero;

- $\quad$ POS - positive small;

- $\quad \mathrm{POM}-$ a positive average;

- $\quad \mathrm{POB}$ - positive big.

Defuzzification or display of fuzzy sets in a single value (the amplitude of pedestal current $I_{m}$ at the controller's output) as the result of aggregation of the fuzzy rules block of in numerical value, is carried out on a center of area method. The output signal of the FL controller received in such a way is multiplied with a signal of an identification unit of external parameters of optimal FCD control system in phase with active current of a circuit that provides synchronization of the FL controller with a circuit and, as a result, the consumption of purely active current for voltage maintenance on CB FCD.

As a result, the received set consisting from 49 rules can be presented in the matrix type by a table 1 . Rules implement the principle of inverted pendulum.

The results of the designed FL-controller' modeling and a comparison with the PI-controller showed that this FL-controller allows to reduce the minimum value of current's total harmonic distortions to $2,5 \%$ in comparison with $5,4 \%$ in case of the PI-controller.

\section{An advanced model of the controller based on a system of neuro-fuzzy inference (ANFIS adaptive neuro-fuzzy inference system)}

As researches showed, the designed system has a number of defects at all the advantages of the received controller. So, for the achievement of preset quality index exceeding those of PI-controllers it appeared that the control system on the basis of fuzzy logic must include the excess quantity of fuzzy rules that is difficult from the point of view of computational capability even modern digital systems. One more essential fault of such controllers is the lack of the systematizing procedures of creation and the analysis of monitoring systems on fuzzy logic. Heuristic and iterative approach (method) to exact set-up of fuzzy rules base and membership functions requires large time expenditures. There are no clear criteria of membership functions' form choice, their steepness, intersection degree, quantization levels of variables and the value of fuzzy rules. Besides, the methods based on fuzzy rules requires large computing costs of a defuzzification, making systems on fuzzy logic difficult and slow that requires the further ways search of operation optimization of designed controller. One of the elimination ways of noted disadvantages authors see the use of adaptive systems neuro-fuzzy inference which combine the advantages of FL and neural networks, providing smoothness of FL controllers regulation and adaptability of artificial neural networks [3].

In this study, the use of adaptive neuro-fuzzy inference system functionally equivalent to Sugeno fuzzy systems is suggested for the elimination of defects. Structurally ANFIS consists of five layers (fig. 3), in each of which the units have various structure and function. The first layer carries out a fuzzification, that is the formation of membership functions fuzzy sets for each of partial optimality criteria. The quantity of units (neurons) is equal to the product of output partial criteria quantity on the total amount of terms used their membership functions [4].

There is a parameters tweaking of a fuzzification of each layer units in the course of ANFIS training. That is parameters $\mathrm{a}, \mathrm{b}$ and $\mathrm{c}$ change. The values of membership functions $\mu i$ are formed at the output of a layer.

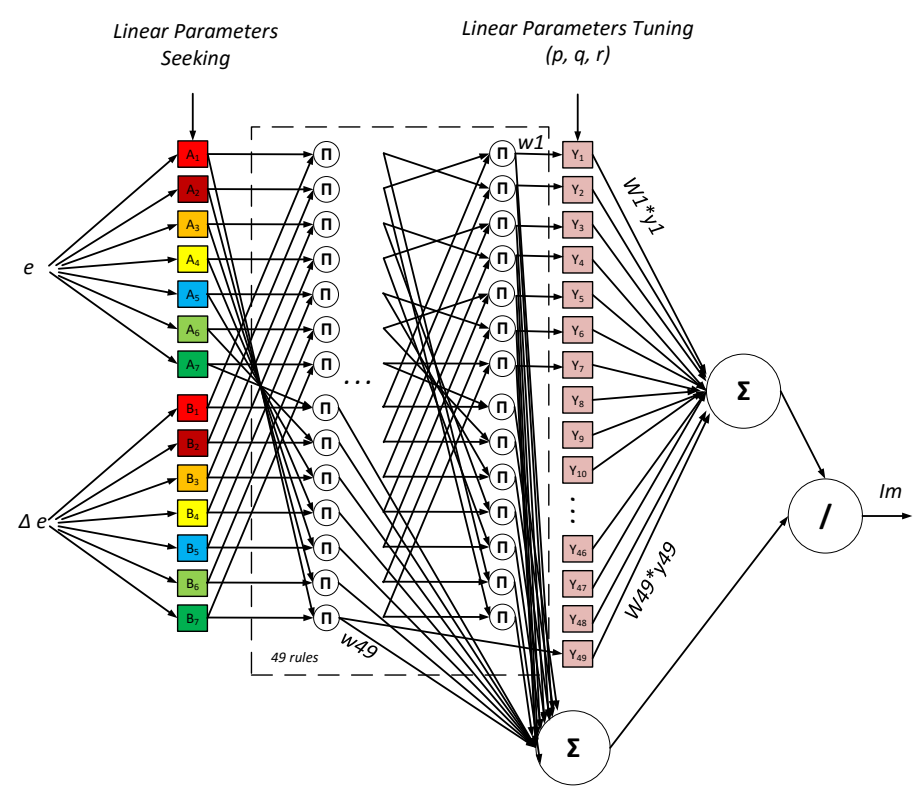

Fig.3. The general structure of adaptive neuro-fuzzy inference system. 
The second layer carries out aggregation, that is determines the degree of the conditions' truth at each rule of fuzzy inference system, forming the value of weight coefficient at the output of each unit $[4,5]$.

$$
w_{i}=\mu_{e_{i}}(e) \cdot \mu_{\Delta e_{j}}(\Delta e)
$$

It means that the neurons of the second layer act as antecedents or hypotheses of fuzzy rules, forming the truth degree of prerequisites $w_{i}$ at the output of layer of each rule of system knowledge base The third layer carries out an activation, that is the determination of truth degree of each aggregated rules of fuzzy production. Here a normalization of rule firing, and the output of $i$ neuron of this layer represents the ratio of truth degree of $i$ rule's prerequisite to the sum of all rules prerequisites' degrees [69]:

$$
\bar{w}_{i}=\frac{w_{i}}{w_{1}+w_{2}+\ldots}
$$

The conclusions of rules are calculated in the fourth layer. The layer has outputs of Takagi-Sugeno type, that is it forms the crisp values determined by linear functions:

$$
\bar{w}_{i} y_{i}=\bar{w}_{i}\left(p_{i} e+q_{i} \Delta e+r_{i}\right)
$$

where $\mathrm{p}, \mathrm{q}$ and $\mathrm{r}$ - the reconfigurable parameters.

Thus, a formation of fuzzy knowledge base tab. 1 quantification on it and implication occurs in layers 2-4.

The aggregation of result received according various rules is carried out in the fifth layer. This layer contains the single neuron which calculates ANFIS output value $[10,5,11]$. For a case of two criteria of $x_{1}$ and $x_{2}$ :

$$
\sum_{i} \bar{w}_{i} y_{i}=\frac{\sum_{i} w_{i} y_{i}}{\sum_{i} w_{i}}=\sum_{i} \bar{w}_{i}\left(p_{i} e+q_{i} \Delta e+r_{i}\right)
$$

The output signals of ANFIS are still the deviation $e$ of voltage on CB FCD from reference value and the change $\Delta e$ this deviation. An output signal is the amplitude value of AFCD reference current.

The training signal is formed by the FL controller suggested above. The essence of the suggested method consists in the fact that the FL-controller used for training of ANFIS uses Gaussian copula membership functions and the rule database presented by table 1. Membership functions of the ANFIS-controller, on the contrary, are realized by triangular functions. The trained under the guidance of the ANFIS FL controller rearranges its structure in such a way that when using triangular membership functions reaches controllers readings with Gaussian copula functions at the reduced computing costs, due to the simple function forms. The difference of ANFIS output signals and the FL controller for a test value series of output signals $e$ and $\Delta e$ is an ANFIS training error, which is counted on each iteration. The training process ends after a lapse of the preset amount of periods, or at the reaching of error training's boundary value $\varepsilon$. The quality of the trained ANFIS is checked by the following series of signals' test values $e$ and $\Delta e$, and also the output signal of the FL-controller. If the training error doesn't exceed preset $\varepsilon$, the structure of ANFIS is considered strict.

The triangular membership functions were used in Sugeno fuzzy systems. The hybrid method of training at which the training occurs in two steps was used for the training of a synthesized ANFIS:

1) Forward pass: the selection of linear parameters $p, q$ and $r$ is carried out at the fixed nonlinear parameters of ANFIS membership functions a (fuzzification layer). That is the values of the reconfigurable Takagi-Sugeno function parameters are selected. The least squares method is used for selection;

2) Backward pass: the best value of the nonlinear parameters comprising the domain functions are searched at the recorded parameters of an output layer (discovered at forward pass) using a method of gradient descent, optimum values. The value of each parameter at the backward pass of training changes to the value

$$
\Delta \alpha_{i}=-\eta \frac{\partial E}{\partial \alpha_{i}}
$$

where 


$$
\eta=-\frac{k}{\sqrt{\sum_{i}\left(\frac{\partial E}{\partial \alpha_{i}}\right)^{2}}}-\text { training coefficient; }
$$

$k-$ step size;

$$
\frac{\partial E}{\partial \alpha_{i}} \text { - the partial derivative of training error according corresponding parameter. }
$$

ANFIS combines the advantages of FL and neural networks, providing the smoothness of regulation of FL controller and the adaptivity of artificial neural networks.

\section{Conclusion}

The suggested controller was modeled in the ANFIS editor of the MATLAB software environment. It was established that the system is trained for 5-7 iterations, without the increase of an training error at the subsequent iterations that evidences about the lack of a network retraining as a result of modeling.

The total harmonious distortions of current in case of ANFIS use according to the simulation data doesn't exceeded 3.4\% that is a little worse than the FL controller with Gaussian membership functions.

The transient characteristic of ANFIS controller are identical to characteristics of the FL controller. Theoretically maximum reached power measure with it reaches the value 0,999 in comparison with the value 0,979 maximum reached in case of PI controller use and 0,9997 for the FL-regulator. When dumping the load of 50\% in 0.001 seconds and double increase for 0.005 seconds the reaching of the new established value of current occurs smoothly, without overcompensation.

The use of the ANFIS controller allows to reduce the maximum voltage deviation on CB. Reaching of the established voltage value on $\mathrm{CB}$ ends within several periods of circuit voltage, and the necessary computing power of the digital system realizing the ANFIS controller decreases in the dozens of times, in comparison with the FL controller with Gaussian copula membership functions.

\section{References}

[1] M. D. Cox and A. Mirbod, "A New Static Var Compensator for an Arc furnace," IEEE Trans. on Power Systems, Vol. PWRS- 1(3): 110-1 19 August 1986.

[2] G. Manchur and CC. Erven, "Development of a Mode1 for Predicting Flicker from Electric Are Fumaces," IEEE Tran. On Power Delivery, Vo1.7, No. 1, pp. 416-426, January 1992.

[3] Zhilenkov A., Chernyi S. Investigation performance of marine equipment with specialized information technology / Procedia Engineering. Vol. 100, 2015, pp. 1247-1252

[4] Chernyi S., Zhilenkov A. Analysis of complex structures of marine systems with attraction methods of neural systems. Metallurgical and Mining Industry, 2015, No. 1 pp. 37-44

[5] Chernyi S., Zhilenkov A. Modeling of complex structures for the ship's power complex using XILINX system. Transport and Telecommunication. 2015. Vol. 16 (1), pp. 73-82

[6] IEEE Working Group on Power System Harmonic, "Bibliography of Power System Harmonics, Part 1 and Ii," Papers 84WM 214-3, IEEE PES Winter Meeting, January 29-Febniary 3, 1984.

[7] IEEE Working Group on Power System Harmonic, "Power System Harmonics: An Overview," XEEE Dans. on Power App. Syst., PAS-102 (8), pp. 2455-2460, Aug. 1983.

[8] B. Singh, K. Al-Haddad, A. Chandra A Review of Active Filters for Power Quality Improvements IEEE Trans. on Indusinal Electronics, 46 (5) (Oct. 1999), pp. 960-971

[9] S.N. Govindarajan, et al. Survey of Harmonic Levels on the Southwestern Electric power Company System IEEE tram. on Power Delivery, 6 (4) (Oct. 1991), pp. 1869-1873

[10] Chernyi S. The implementation of technology of multi-user client-server applications for systems of decision making support / Metallurgical and Mining Industry. ( 2015). Vol. 3. pp. 60- 65

[11] Kramar Vadim, Influence of Stress-Corrosion Fractures on Potential of Ship-Building Metals in the Sea Water / Vadim Kramar, Veronika Dushko, Anna Rodkina, Anastasiia Zaiets // Annals of DAAAM for 2014, Volume 25, No.1, ISSN 2304-1382 \& Proceedings of the 25th DAAAM International Symposium on Intelligent Manufacturing and Automation, Volume 100, ISSN 1877-7058, Edited by Branko Katalinic, ELSEVIER, 2015. - P. 1068-1074. 